\title{
Evaluation Du Système D’hygiène Dans Les Locaux Des Restaurationsscolaires, À La Région d'El Gharb Cherardabnihssen, Par Rapport Auxexigences Normatives Internationales
}

\author{
S. El Ghaza \\ Y. Chbab \\ E. Bakrim \\ B. Zaadoud \\ A. Chaouch
}

Laboratoire de Biotechnologie, Environnement et Qualité, Département de

Biologie, Faculté des Sciences, Univ Ibn Tofaïl, Kénitra, Maroc

doi: 10.19044/esj.2016.v13n2p243 URL:http://dx.doi.org/10.19044/esj.2016.v13n2p243

\begin{abstract}
The implementation of hygiene system in the school catering is based on the application of good hygienic practice and regulations of food safety (security). These measures of hygiene contribute absolutely, in the elimination of the potential food risks susceptible to be at the origin of several diseases.

This article presents the results of a study carried out to evaluate the degree of the respect for the sanitary conditions at the level of three school restaurants. The result of our study aims to determine the level of compliance of their hygienic systems to international hygienic standards.

The rates of conformity of the sites of the study were of the order of 33, 60 $\%, 33,00 \%$ and of $48,58 \%$ respectively at the level of the establishment $\mathrm{n}^{\circ} 1, \mathrm{n}^{\circ} 2$ and $\mathrm{n}^{\circ} 3$.

The recorded results show apparently, the level of conformity with regard to the standards. In front of this situation, it is essential to plan and to implement relevant actions of improvement and to engage ways and resources to eliminate the noticed gaps and increase the level of conformity.
\end{abstract}

Keywords:Hygiene, risk, standard compliance, restoration

\section{Résumé}

La mise en place d'un système d'hygiène dans la restauration scolaire est basée sur l'application des bonnes pratiques d'hygiène et les règles de 
sécurité alimentaire. Ces dispositions d'hygiène contribuent absolument, dans l'élimination des risques alimentaires potentiels susceptibles d'être à l'origine de plusieurs maladies.

Cet article présente les résultats d’une étude réalisée pour évaluer le degré du respect des conditions d'hygiène au niveau de trois restaurants scolaires.Les résultats de notre étude sont évalués par rapport aux critères techniques des référentiels déterminant les exigences relatives à la sécurité et à la qualité des denrées alimentaires internationaux.

Les taux de conformité des sites de l'étude ont été de l'ordre de 33,60\%, $33,00 \%$ et de $48,58 \%$ respectivement au niveau de l'établissement $n^{\circ} 1, n^{\circ} 2$ et $n^{\circ} 3$.

Les résultats enregistrés montrent visiblement, le niveau de conformité par rapport aux normes. Devant cette situation, il est indispensable de planifier et mettre en œuvre des actions d'amélioration pertinentes et engager des moyens et des ressources pour éliminer les écarts constatés et augmenter le niveau de conformité.

Mots-clés : Hygiène, risque, norme, conformité, restauration

\section{Introduction}

L'alimentation des enfants à l'âge scolaire est une condition essentielle pour leur croissance physique, mentale, leur développement psychomoteur et leurs capacités d'apprentissage. Elle constitue un déterminant majeur de la santé(Ministère de la santé, 2011). La stratégie nationale de santé scolaire et universitaire mise en place en 2011 vise, entre autre, à faire des élèves des citoyens ayant un mode de vie sain et dotés des compétences nécessaires pour pouvoir être responsables de leur santé. Elle a pour objectifs de contribuer à réduire les comportements à risques, d'augmenter le recours aux services de santé et d'améliorer les conditions d'hygiène et de sécurité au sein des établissements de l'enseignement (Projet de PNAS, 2013).

Les locaux des restaurations scolairessont des lieux privilégiés pour manipuler des repas de qualité dépourvus des dangers chimiques, biologiques et physiques.Les conditions de préparation des menus sont des paramètres primordiauxet essentiels pour assurer la salubrité des repas. En effet, l'application des règles d'hygiène permet de prévenir des toxiinfections alimentaires collectives, lutter contre les sources de contamination et réduire les voies de transmission.

Notre étude s'inscrit dans un contexte d'évaluation du système d'hygiène par rapport aux exigences des normes internationales, en vue de faire un état des lieux pour améliorer le niveau de respect et d'application des règles d'hygiène relatives aux locaux, aux personnels, aux équipements 
et matériels,à la chaine de préparation des aliments etau système de traçabilité ...etc.

\section{Objectif de l'étude}

L’objectif de notre étude est d'évaluer et de vérifier le niveau de conformité des conditions d'hygiène au niveaudes restaurations des trois établissements, par rapport aux critères techniquesdes référentiels. Les résultats de cette évaluation nous permettront de mettre en évidence les nonconformités et les anomalies réelles, et déterminer par la suite les actions correctives à mettre en œuvre pour améliorer la situation existante, notamment en matière de propreté et d'hygiène.

\section{Champs d'application de l'étude}

Notre étude d’évaluation a été appliquée au niveau des restaurations de trois établissements à la région d'El Gharb-Cherarda-Bni-Hssenauxquels nous avons attribué des codes à titre indicatif.

- $\quad$ Etablissement $n^{\circ} 1: \mathrm{SE} 1$

- $\quad$ Etablissement $n^{\circ} 2$ : SE2

- $\quad$ Etablissement $n^{\circ} 3$ : SE3

Cette évaluation a été effectuée au niveau des locaux des restaurations : la cuisine, le réfectoire, la zone de stockage, les toilettes, les équipements et le matériel utilisés dans lechaine de préparation des repas.

D’autres dimensions ont été évaluées : le personnel exerçant les activités de cuisson,les méthodes de nettoyageet de désinfection et le transport.

\section{Normes et référentielsutilisés}

L’application des règles d’hygiène vise essentiellement, la prévention des maladies transmissibles en collectivité et la lutte contre les sources de contamination et réduire les moyens de transmission(C.S.H.P.F, 2010).

La mise en place d'un système d'hygiène nécessite l'application des normes et standards en vigueur. La culture qualité développée chez le consommateur, a eu un rôle prédominant dans le développement du concept de la sécurité des aliments. La réglementation européenne, par l'intermédiaire du règlement 178/2002 et du Paquet Hygiène, préconise la mise en œuvre des normes hygiéniques qui ont une répercussion importante sur les industries agroalimentaires bretonnes et françaises (J. R. GEOFFROY et J. CHESNAIS, 2006).

La série des normes et standards adoptés dans notre évaluation est composée de :

\section{- $\quad$ La norme ISO 22000 ;}

- $\quad$ La norme BRC ; 
- $\quad$ La norme IFS ;

- $\quad$ Le Codex Alimentarius.

Le guide des bonnes pratiques, le programme préalables et certains documents de référence de l'ONSSA (l'Office National, de la SécuritéSanitaire Alimentaire) ont été utilisés pour enrichir le support d’évaluation.

\section{La norme ISO 22000version 2006}

La norme ISO 22000 spécifie les exigences d'un système de management de la sécurité des denrées alimentaires. L’application de cette norme au niveau des processus de préparation des repas aident l'établissement à donner confiance aux parties intéressées (élèves, enseignants, association des parents d'élève...).

Ce système a initialement été mis au point dans les années 1960 pour la production de denrées alimentaires saines et sûres dans le cadre du programme spatial américain(ONEAA, 1998). Le système d'analyse des risques - points critiques pour leur maîtrise (HACCP), est un outil que les entreprises du secteur agroalimentaire utilisent pour garantir la sécurité sanitaire des aliments (Norme ISO 22000, 2006).

Reposant sur la prévention et sur l'analyse des risques, ce système permet d'identifier les points critiques pour la maîtrise (CCP) des risques physiques (ex. verre), chimiques (ex. pesticides) et microbiologiques (ex. bactéries responsables d'intoxications alimentaires), avant qu'ils ne compromettent la sécurité sanitaire des aliments(ONEAA, 1998). Selon la législation, toutes les entreprises agroalimentaires européennes doivent instaurer et appliquer des procédures s'appuyant sur les principes du système HACCP(Journal Officiel de l’Union européenne, 2004).

\section{Autres référentiels}

\section{BRC : British Retail Consortium}

Le B.R.C est un référentiel qui défini les critères de sécurité, de qualité et de production qui doivent être mis en place dans une organisation qui fabrique et prépare des denrées alimentaires, pour satisfaire les obligations en matière de respect de la législation et de protection du consommateur. C'est un outil qui vise l'évaluation du système de management de la sécurité des denréesalimentaire de type HACCP (Norme BRC, 2005).

\section{IFS : International Food Standards}

La norme de l'IFS a été développée par les distributeurs, les industriels, les services alimentaires et les organismes de certification. Cette norme est le seul référentiel de transformation alimentaire à avoir impliqué 
de manière équivalente par toutes les parties prenantes de la chaîne alimentaire (Norme IFS Food, 2011).

\section{Codex Alimentarius}

Le Codex Alimentarius, ou code alimentaire, est devenu la référence mondiale qui fait autorité pour les consommateurs, les producteurs et les transformateurs de denrées alimentaires, les organismes nationaux de contrôle des aliments et le commerce international des produits alimentaires [10]. Le Codex Alimentarius comporte des directives très variées visant à protéger les consommateurs, sur des sujets très divers comme l'établissement et l'application de critères microbiologiques pour les aliments.

L’objectif principal de l'application des normes du code alimentaire dans la restauration scolaire est la protection de la santé des consommateurs. Le respect des directives et des recommandations du Codex Alimentarius est nécessaire pour minimiser les risques de contamination à chaque étape de la chaine de préparation et développer la culture de l’hygiène et de la sécurité alimentaire.

\section{Dimensions évaluées Personnel}

La formation continue du personnel opérant dans les restaurations scolaires, sur les règles et les bonnes pratiques d'hygiène, est désormais une obligation. Chaque établissement a l'obligation d'élaborer annuellement un plan de formation global du personnel de restauration, notamment en matière d’hygiène alimentaire (Norme IFS Food, 2011). La sensibilisation du personnel en la matière aurait des conséquences positives pour assurer la sécurité alimentaires et éliminer les sources de contamination.

Le suivi médical du personnel vise à protéger d'une part, la santé du personnel et d'autre part, celle du consommateur. La surveillance médicale des personnels, la reconnaissance de l'aptitude ou de l'inaptitude à un poste de travail révèle du médecin du travail ou du médecin de prévention (Norme BRC, 2005).

Des procédures de déclaration des maladies sont des outils documentées pour que les employés, signalent les maladies ou états infectieux dont ils sont atteints ou avec lesquels ils peuvent avoir été en contact.

\section{Construction des locaux}

Les locaux et les installations des restaurations scolaires doivent être conçus, construits, entretenus pour offrir des espaces de travail et de stockage suffisants et permettre que toutes les opérations soient réalisées correctement dans des conditions d'hygiène sûres. 
La construction de la cuisine, des réfectoires et des toilettes doit être effectuée en respectant les règles d'hygiène. Les murs, les plafonds et les sols doivent être conçus, construits, finis et entretenus de manière à éviter l'accumulation de saletés, limiter la condensation et le développement des moisissures et faciliter leur nettoyage.

Les zones de stockage des aliments doivent être construites en respectant les mesures d'hygiène pour assurer des conditions favorables en terme de température, d'humidité, d'éclairage ...etc. Le contrôle permanent de ces paramètres permettra de prévenir la multiplication des microorganismes (moisissures et bactéries) et lutter contre les vecteurs et les nuisibles.

\section{Equipements et maintenance}

Les équipements et les machines, incluant tous les accessoires et les instruments de travail doivent être convenablement conçu pour l'usage qui leur est destiné et être maintenus dans des conditions permettant de limiter les risques de contamination des produits. A la fin de chaque action de maintenance, la machine et l'équipement doivent être propres et exempts de danger de contamination. Un planning de d'entretien préventif doit être élaboré et appliqué par des responsables qualifiés et formés.

\section{Matériels et méthode}

\section{- $\quad$ Adaptation des critères du référentiel d'évaluation}

Une revue générale sur les référentiels et standards d'hygiène,citésprécédemment,a été réalisée pour identifier des critères d'évaluation qui sont adaptés aux spécificités des restaurations scolaires.

Nous avons établi un questionnaire contenant plus de 200 critères, relatifs à différentes dimensions d'hygiène. Le tableau représenté ci-dessous présente le modèle de la grille d'évaluation utilisée dans notre étude.

Tableau $n^{\circ} 1$ : Modèle de la grille d'évaluation

\begin{tabular}{|c|c|c|c|c|}
\hline \multirow{2}{*}{ Dimensions } & \multirow{2}{*}{ Critères d'évaluation } & \multicolumn{3}{|c|}{ Evaluation } \\
\hline & & $\mathrm{C}^{*}$ & $\mathrm{PC}^{* *}$ & $\mathrm{NC}^{* * *}$ \\
\hline \multirow{5}{*}{$\begin{array}{l}\text { Hygiène du } \\
\text { personnel }\end{array}$} & \multicolumn{4}{|c|}{$\begin{array}{l}\text { Les mains doivent être lavées efficacement et fréquemment, en } \\
\text { particulier : }\end{array}$} \\
\hline & A la reprise du travail & & & \\
\hline & A la sortie des toilettes & & & \\
\hline & $\begin{array}{l}\text { Après manipulation de cartons, matériels sales, } \\
\text { poubelles, déchets, produits chimiques... }\end{array}$ & & & \\
\hline & $\begin{array}{l}\text { Le port des bijoux doit être interdit, à } \\
\text { l'exception des alliances simples, des bracelets } \\
\text { de mariage et des boucles d'oreille en anneaux } \\
\text { fermés (boucle continue). }\end{array}$ & & & \\
\hline Suivi médical & Un contrôle médical doit être réalisé & & & \\
\hline
\end{tabular}




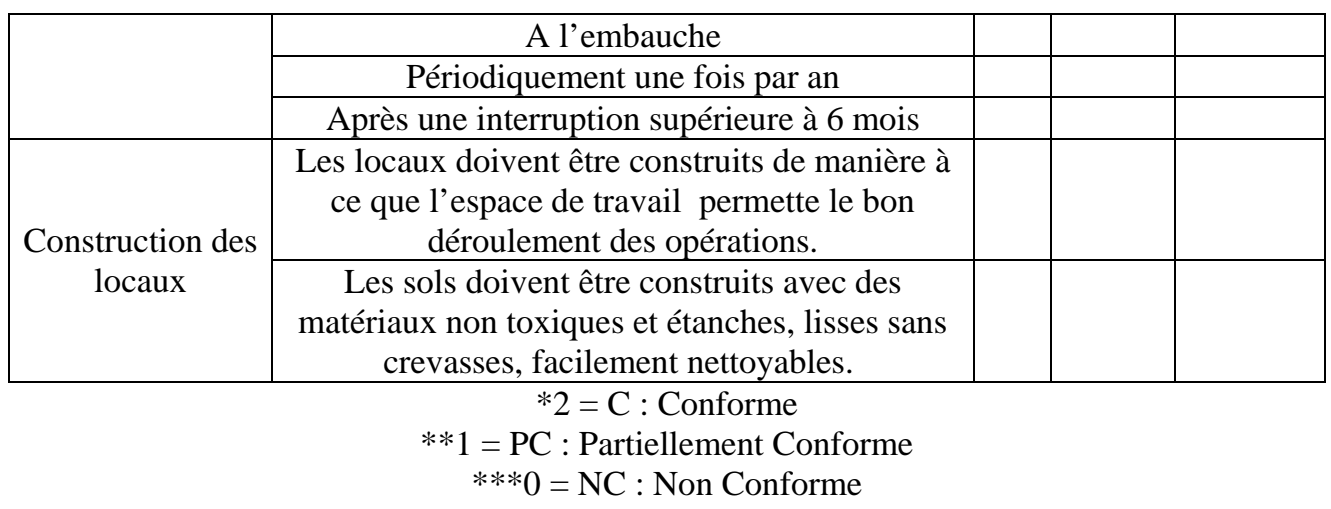

\section{- $\quad$ Planification et réalisation des visites d'évaluation}

L'évaluation de la situation de référence a été programmée au niveau des trois établissements. Au cours de notre processus d'évaluation, nous avons adoptés plusieurs méthodes pour collecter des informations relatives à l'application des règles d'hygiène au niveau des sites de l'étude.

Des entretiens ont été effectués avec les responsables des opérations de restauration et le personnel manipulant les activités de préparation des repas ainsi que les femmes de nettoyage. Aussi, cette étude a été à la base des observations pour visualiser réellement le respect des bonnes pratiques d'hygiène au cours des activités de préparation, de lavage des équipements et au cours des opérations de nettoyage.

Les réponses collectées ont été renseignées sur des grilles d'évaluation.

Par la suite nous avons défini une méthode de cotation pour calculer les taux de conformité et de non-conformité et de tirer des constations sur le niveau du respect des critères d'hygiène définis.

\section{- Méthode de cotation}

Au niveau de la grille, nous avons attribué pour chaque réponse un coefficient de 0,1 et 2 pour quantifier et présenter les résultats sous forme de graphique.

Nous avons attribué la note (2) aux critères conformes, la note (1) aux critères partiellement conformes et (0) pour les critères non conformes.

Les taux sont calculés par les formules suivantes :

\section{Le taux de conformité :}

$$
\% \mathbf{C}=\frac{(\text { Nombre des critères notés } * 2)}{(\text { Nombre total des critères } * 2)} * 100
$$


Le taux de conformité partielle :

$$
\% \mathbf{C P}=\frac{(\text { Nombre des critères notés } * 1)}{(\text { Nombre total des critères } * 2)} * 100
$$

Le taux de non-conformité est calculé par la formule suivante :

$$
\% \mathrm{NC}=100-(\% \mathrm{C}+\% \mathrm{CP})
$$

\section{Résultats et Discussion}

\section{- $\quad$ Par dimension de l'établissement (SE1)}

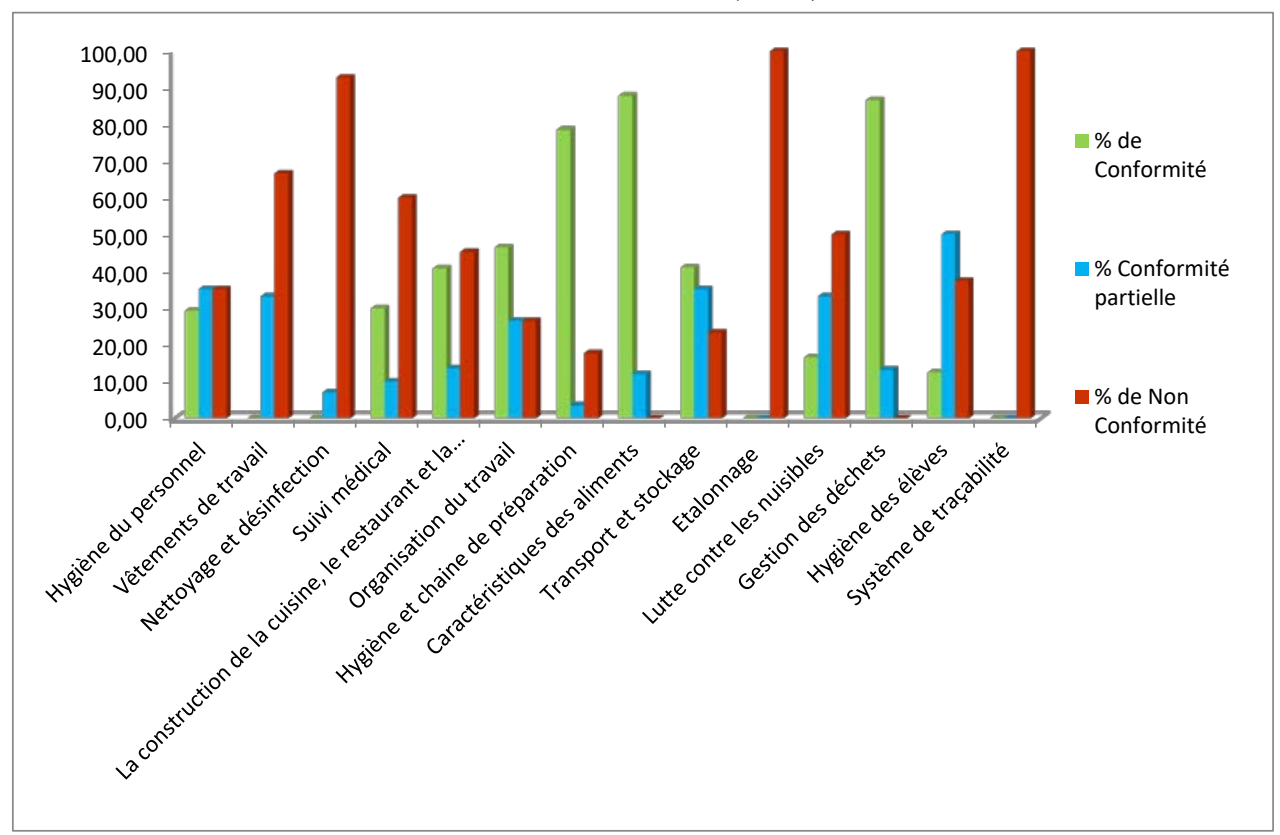

Figure $\mathbf{n}^{\circ} \mathbf{1}$ : Résultats d'évaluation par dimension des sites de l'établissement $\mathrm{n}^{\circ} 1$

\section{Commentaire}

Les résultats d'évaluation des dimensions d'hygiène de l'établissement (SE1), représentés sur la figure $n^{\circ} 1$,montrent que les règles d'hygiène relatives à la gestion des déchets, aux caractéristiques des aliments et à la chaine de préparation représentent des taux de conformités supérieurs à $80 \%$. En revanche nous avons constaté des taux de nonconformité supérieurs à $90 \%$ pour les dimensions relatives aux activités de nettoyage et de désinfection, à l'étalonnage et au système de traçabilité. Les règles relatives auxvêtements de travail et au suivi médical ont un taux de non-conformité supérieure à $60 \%$. 
- $\quad$ Par dimension de l'établissement (SE2)

Figure $\mathbf{n}^{\circ} \mathbf{2}$ : Résultats dévaluation par dimension des sites de l'établissement $n^{\circ} 2$

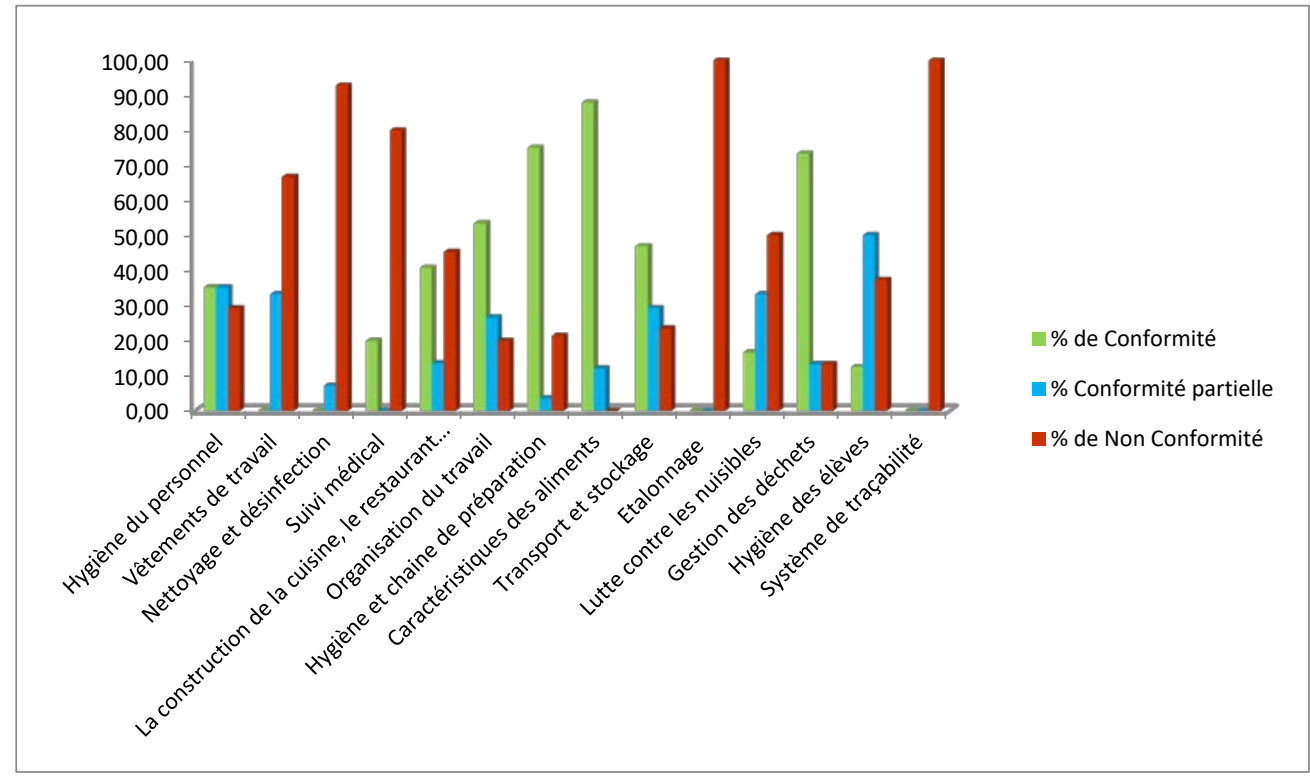

\section{Commentaire}

Pourle deuxième établissement (SE2), la représentation graphique montre que les résultats d'évaluation sont identiques à ceux de l'établissement $n^{\circ} 1.75 \%$ des non conformitésa été enregistrée pour la gestion des déchets, aux caractéristiques des aliments et à la chaine de préparation. Un écart de $100 \%$ a été calculé pour ce qui est du système de traçabilité et de l'étalonnage. Le niveau de non-conformité des critères relatifs aux vêtements de travail, au suivi médical et aux opérations de nettoyage et de désinfection a été estimé à une valeur $80 \%$.

\section{- $\quad$ Par dimension de l'établissement (SE3)}

Figure $\mathbf{n}^{\circ} \mathbf{3}$ : Résultats dévaluation par dimension des sites de l'établissement $\mathrm{n}^{\circ} 3$

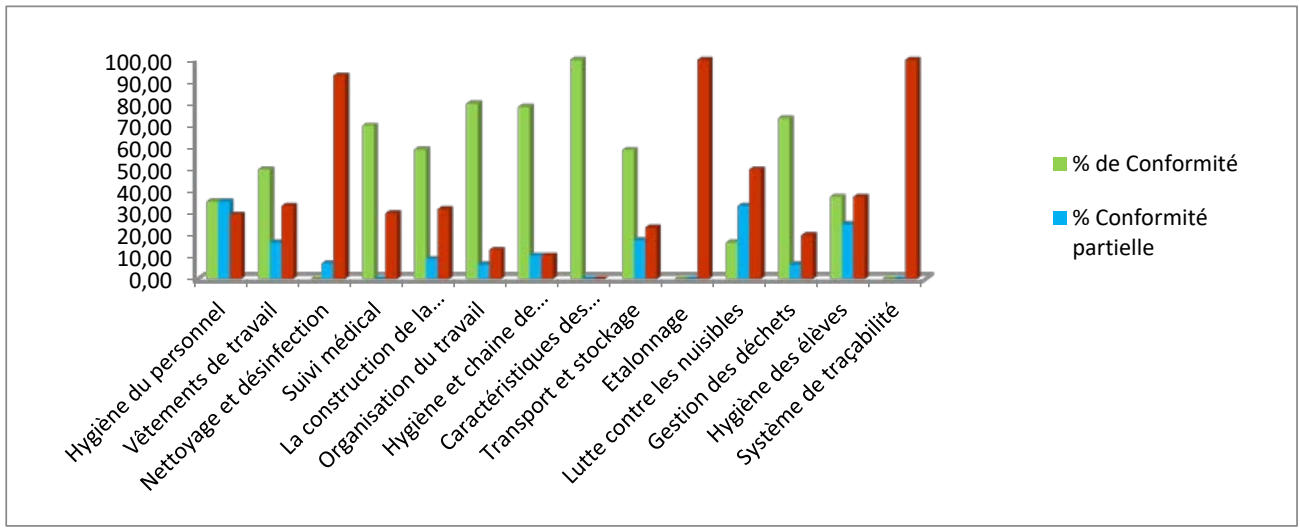




\section{Commentaire}

Par rapport aux sites d'étude des deux établissements précédents, un taux de conformité de $100 \%$ a été enregistré au niveau des règles appliquées aux caractéristiques des aliments. D'autres critères relatifs à l'organisation du travail et à la chaine de préparation ont un taux de conformité de $80 \%$. Les exigences de la gestion des déchets et le suivi médical ont été respectées à un taux de 70\%. Les taux de non-conformité les plus élevés ont été calculés pour le système de traçabilité et de l'étalonnage (100\%) et le nettoyage et désinfection (95\%).

\section{- Résultats par établissement}

Figure $\mathbf{n}^{\circ} 4$ : Résultats de l'établissement ES1

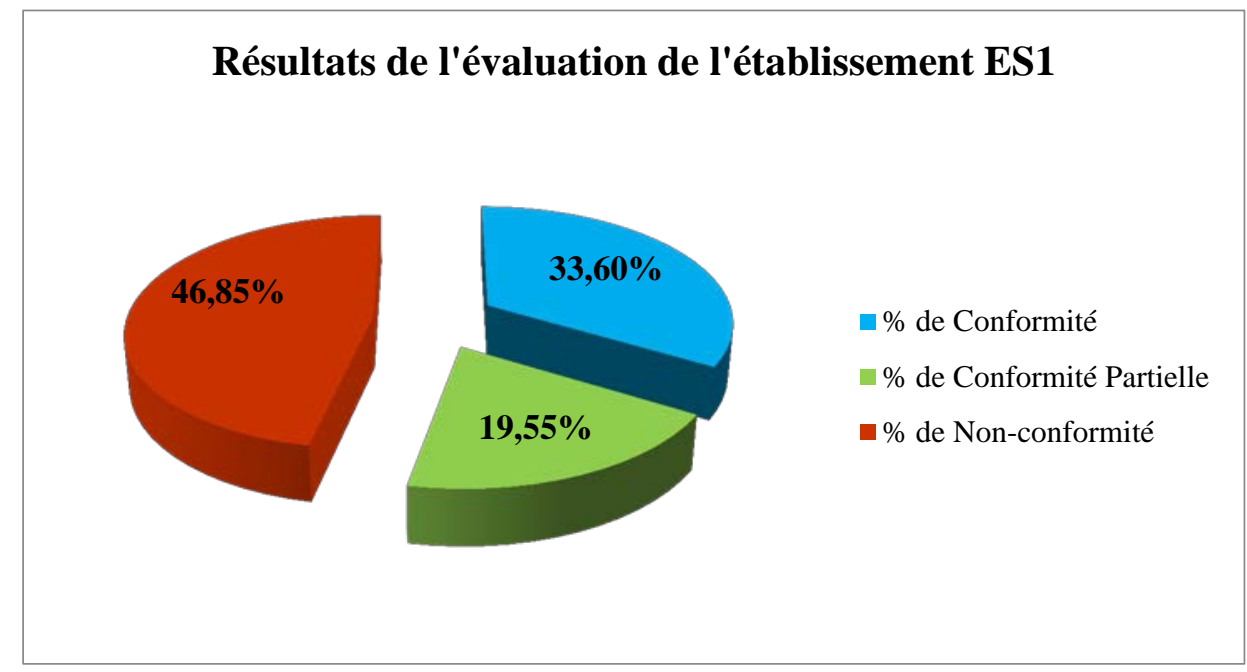

L'analyse quantitative de la situation existante nous a permis de constater un taux moyen de conformité de 33,60\%, une valeur de $19,55 \%$ de conformité partielle et une valeur moyenne de $46,85 \%$ de non-conformité.

Figure $\mathbf{n}^{\circ} 5$ : Résultats de l’établissement ES2

\section{Résultats de l'évaluation de l'établissement ES2}
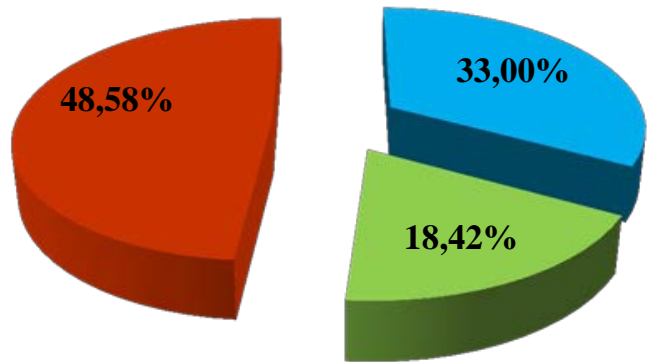
- \% de Conformité
\% de Conformité
Partielle


Presque les mêmes valeurs ont été calculées pour le deuxième établissement : 33,00\% de conformité, 18,42\% partiellement conforme et 48,58\% de non-conformité.

\section{Résultats de l'évaluation de l'établissement ES3}
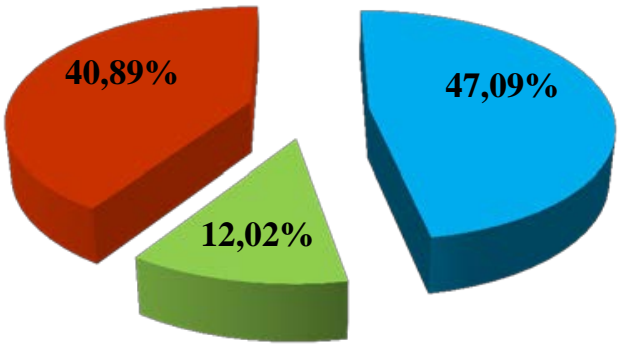

\% de Conformité

\% de Conformité

Partielle

Figure $\mathbf{n}^{\circ} 6$ : Résultats de l'établissement ES3

$\mathrm{Au}$ niveau du troisième site, nous avons calculé un taux de conformité de 48,58\% des critères d'hygiène. Une valeur moyenne de $12,02 \%$ de critères partiellement conformes et $40,89 \%$ de non-conformité.

En comparant ces résultats avec ceux des deux sites précédentes, on remarque une nette amélioration au niveau de l'application des règles d'hygiène particulièrement celles relatives au suivi médical, à l'organisation du travail, à la chaine de préparation, aux caractéristiques des aliments et à la gestion des déchets.

\section{- $\quad$ Discussion des résultats}

La lecture des résultats figurés sur les trois graphiques a permet de constater des taux de conformité qui varie d'un établissement à un autre et de quantifier le degré du respect de l'application des critères d'hygiène dans les sites choisis.

On peut dire que ces constatations sont dues principalement au manque de sensibilisation des décideurs en matière d'hygiène et l'absence des formations du personnel sur les règles d'hygiène et sur les techniques de lavage des mains, lavage des vêtements de travail et sur l'organisation des activités de restauration et le circuit d'acheminement des aliments.

Les conditions et le rangement de stockage et les modalités de transport des aliments doivent être respectées et les locaux de restauration doivent être construits en tenant compte l'effectif du personnel et des élèves afin d'éviter la contamination croisée et faciliter les méthodes de nettoyage. 
La nécessité d'établir des procédures de travail, des fiches de contrôles, des plannings de travail sont des actions qui permettent de constituer un système de traçabilité pour assurer et garantir une application parfaite et maitrisée des instructions et des consignes relatives aux bonnes pratiques et aux règles d'hygiène.

Pour remédier à cette situation, un plan d'amélioration doit être établi et mis en œuvre pour corriger les écarts détectés. La mobilisation des ressources et la responsabilisation du personnel constituent des facteurs clés de réussite de la mise en place des actions correctives adaptées et efficacespour améliorer le niveau de conformité et diffuser une culture d'hygiène dans les sites d'études des établissements.

\section{Conclusion}

Les résultats de l'évaluation de l'application des règles d'hygiène au niveau des trois établissements ont montré des taux de non-conformités très élevées par rapport aux normes et standards.

L'application des bonnes pratiques d'hygiène, conformément aux standards internationaux, dans les activités de restauration favorisera un environnement sain et dépourvu des sources de contamination du personnel et des élèves. La manipulation des repas en respectant les règles d'hygiène permettra au personnel de préparer des repas salubres et de qualité sans risque.

Un plan des actions correctives devrait être établi et validé pour corriger les non conformités constatées et améliorer le système d’hygiène.

\section{References:}

1. La Stratégie Nationale de la Nutrition, 2011-2019, p8

2. Développement des capacités et appui au programme national d'alimentation scolaire, 1er décembre 2012 - 31 décembre 2013, p8 ;

3. La Stratégie Nationale de la Nutrition, 2011-2019, p15 et 16

4. Guide des conduites à tenir en cas de maladie transmissible dans une collectivité d'enfants,

5. Mesures d'hygiène en collectivité, 29 décembre 2010

6. J. R. GEOFFROY et J. CHESNAIS, Comparaison des référentielsBRC - IFS - ISO 22000, Janvier 2006, p3

7. L'Organisation des Nations Unies pour l'agriculture et l'alimentation (1998). Systèmes de qualité et de sécurité sanitaire des aliments Manuel de formation sur l'hygiène alimentaire et le Système d'analyse des risques - points critiques pour leur maîtrise (HACCP).

8. Règlement (CE) $N^{\circ}$ 852/2004 du Parlement Européen et du Conseil du 29 avril 2004 relatif à l'hygiène des denrées alimentaires :http://eur- 
lex.europa.eu/LexUriServ/LexUriServ.do?uri=OJ:L:2004:139:0001:0 054:fr:PDF

9. Norme ISO 22000, système de Management et de la sécurité alimentaire, 2005

10. Norme ISO 22000, système de Management et de la sécurité alimentaire, 2005

11. Norme (BRC), RritichRetal Consortium, version 2005

12. Nouveautés IFS- IFS Food 6, Octobre 2011

13. Norme (BRC), RritichRetal Consortium, version 2005 